\title{
What is the meaning of the concept 'consistency' in (chemical) measurement?
}

\author{
Paul De Bièvre
}

Published online: 15 March 2013

(C) Springer-Verlag Berlin Heidelberg 2013

The use of the concept 'consistency' is on the rise in the context 'measurement:' it can be found more and more in "metrological" documents and in the literature. "Consistency" was already used in early, very metrological discussions, for example, evaluations of (fundamental) constants, as obvious from a Panel Discussion in 1970 at the National Bureau of Standards in Gaithersburg (USA): "Should Least-Squares Adjustments of the Fundamental Constants be abolished?" reported in [1]. In this Panel Discussion, even an indirectly explained meaning can be found: "inconsistent" is understood to mean "incompatible" or "discrepant."

The range of meanings of the concept 'consistency' in daily language is very extensive: think of its use, not only in the measurement context, but also in describing a material property (of concrete, or of tissue of living organisms) or in - of all things-politics ("is a politician consistent in what he says?"). It also seems to be used as synonym of 'compatibility' or indicating 'conformity.'

A sounding by e-mail of a (admittedly small) number of people interested in international understanding of necessity requiring a translation of terms, revealed the following spectrum of opinions about 'consistency:'

Absence or freedom from contradictions; 'measurement consistency' is put more or less on equal footing with 'metrological compatibility' as defined in VIM (entry 2.47 in [2]); it can also mean general agreement, conformity;

Disclaimer The author is a member of the Joint Committee on Guides for Metrology (JCGM), Working Group 2 (VIM). The opinions expressed in this Column do not necessarily represent the view of the Working Group or of ACQUAL.

P. De Bièvre $(\square)$

Kasterlee, Belgium

e-mail: paul.de.bievre@skynet.be "consistent" is considered to mean "reproducible;" "consistency' could be defined as a 'notion.'

Sometimes, the problem is tackled in the form of questions: is 'compatibility' a more "egalitarian" relationship than 'consistency'? is 'consistency' the quality of behaving in the same way? and is being consistent the same as being coherent? Last but not least, a definition of 'consistency' is attempted by defining "inconsistency" such as is the case in two or more incompatible beliefs; or when discrepant data are inconsistent, all cannot be true, but all can be false or wrong. Closing question: is consistency the concept, while compatibility is a measure of it?

Following the above limited sounding experiment among a few persons, it seems that 'consistency' is an important requirement in any statement, judgment, or action, but ambiguous in its meaning. Yet, it seems clear that, at least in the field of measurement, we need to understand 'consistency' in such a way that it has the same meaning for all involved in that field. In particular, the statements made in the chemical literature in general, as well as in the frame of the ongoing discussion on the redefinitions of the SI units, there seems to be a need to define both 'metrological consistency' or 'measurement consistency' as a (subordinate) concept as well as 'consistency' as a (superordinate) concept. In other words, there even seems a need to define 'consistency' for use in discussions in other languages for discussion partners to understand what they are talking about.

We could not agree more.

Hence, we should embark on an attempt to define 'consistency' in and after an open debate. We thereby are facing the problem of "language relativism:" when attempting to describe something, we are often in search of suitable terms (words), whereas we should first look for the concepts and their definition (labeled by terms) which then 
enable to translate the label, that is, the term. Only then do we understand what is intended to be translated.

As it seems to be a basic and general concept with associated term, should not we prepare a definition as in the present VIM [2] a definition of 'consistency' is conspicuously absent?

Enough reason to start spreading awareness about the absence of an appropriate definition of 'consistency' in the field of (chemical) measurement. That is the purpose of this Column. After an open debate, we may consider conceiving a definition.

I am greatly indebted to G Price (AU), G Meinrath (DE), F Pavese (IT), B Hibbert (AU), and R Dybkaer (DK), whom I found prepared to deliver a considered opinion in the above (limited) sounding experiment, either of their own, or obtained from their knowledge of authoritative thinkers in the literature (e.g., Aristotle, G W Leibniz, and C F von Weizsäcker,).

As usual, any comment, question, or amendment is welcome, preferably as a contribution to the Discussion Forum of this Journal.
They can also be addressed to the author of this Column for assembling and comparing them for one of the next Columns. Hopefully, that could lead to conclusions.

\section{References}

1. National Bureau of Standards (1970), NBS Special Publication no. 300. Therein: Bender PL, Handling of discrepant data in evaluations of fundamental Constants, pp 493-494; Cohen ER, In defense of least-squares adjustments, pp 491-492; Thomsen J, Some aspects of least squares adjustments of constants, pp 503-505; Eisenhart Ch, Contribution to panel discussion on adjustments of the fundamental constants, pp 509-517; Taylor BN, Comments on the adjustments of the constants, pp 495-498

2. BIPM, IEC, IFCC, ILAC, IUPAC, IUPAP, ISO, OIML (2008/ 2012), The international vocabulary of metrology-Basic and general concepts and associated terms (VIM), edn 3, JCGM at http://www.bipm.org/vim 